\title{
Efficient Layout Design using Transmission Gate in 45nm Technology
}

\author{
Badal Nishant, Meenakshi, Rajesh Mehra
}

\begin{abstract}
XNOR-XOR gates are generally used in arithmetic and logic circuits. This paper presents the performance comparison analysis for the combination of XNOR-XOR circuit by using transmission gate. The transmission gate has consumed only 10 transistors as compared to 16 transistors in case of conventional design. The performance of the simulated circuit has been evaluated and compared in terms of propagation delay, power \& area consumption using $45 \mathrm{~nm}$ technology. It can be observed from the simulated results that proposed circuit consume $63.97 \%$ less area and $90.66 \%$ less power as compared to existing design. The speed performance of proposed design is also improved by $\mathbf{8 5 . 3 8 \%}$ to provide high performance solution for VLSI applications.
\end{abstract}

Index Terms - CMOS, Transmission gate, VLSI, CMOS Technology.

\section{INTRODUCTION}

In the evolution of the integrated circuit towards high frequency with integration density, the concern issues are power, less area and minimum delay with high speed. XNOR and XOR gate is used in several different circuits like an arithmetic circuit, error detection, and code converter. The working of complex logic circuits is depending on the XOR-XNOR circuits [1]. Speed of operations depends on the largest critical paths and number of transistors are used in circuits. [2] In this research paper, we design XOR-XNOR gate using the transmission gate with CMOS inverter circuit $45 \mathrm{~nm}$ technology. Using transmission gate, it covers less area compared to 16 transistors XNOR-XOR with CMOS circuit as well as less power with a smaller delay. There are 2 types of MOS the NMOS and PMOS. Where NMOS transistor provides the "LOW" signal good, but poor for "HIGH" signal. Similar as in PMOS transistor which provides the "LOW" signal completely but poor for "HIGH" signal. So, the CMOS transistor is used. CMOS transistor is combination of PMOS and NMOS transistor which gives good output voltage swing. The design analysis is required for XNORXOR circuit with less delay as well as less power consumption in a critical path [3]-[7]. To reduce power consumption, implementations of a circuit have been considered as a good solution for many applications. [8]

In this research paper, we use circuit of XOR-XNOR gate using transmission gates with CMOS inverter. The advantage of the transmission gate has to provide higher

Badal Nishant, PG Scholars, ECE Department, NITTTR, Sector - 26, Chandigarh, 160001, India

Meenakshi, PG Scholars, ECE Department, NITTTR, Sector - 26, Chandigarh, 160001, India

Rajesh Mehra, Professor, ECE Department, NITTTR, Sector - 26, Chandigarh, 160001, India speed and lower power consumption. This paper is organized in V sections as follows: in Section I introduction, Section II Transmission gate with CMOS inverter is explained, the designing of XNOR-XOR gate using transmission gate and with CMOS explained in section III, in section IV the simulation results and layout design analysis are given and discussed for both $65 \mathrm{~nm}$ and $45 \mathrm{~nm}$ technologies. The evaluation and comparison for transmission gate and existing designs with proposed design are carried out. Finally, conclusions are drawn in section $\mathrm{V}$.

\section{TRANSMISSION GATE LOGIC}

A transmission gate (TG) is which conduct in both directions by a control signal with almost any voltage potential, it is similar to a relay. It is a CMOS-based switch, in which NMOS passes a strong zero and PMOS passes strong one. Both NMOS and PMOS work simultaneously. NMOS and PMOS device gives mediocre performance when transmitting a logic signal. The NMOS deprave the logic level "1" while the PMOS deprave the logic level " 0 ". Thus, an ideal transmission gate can be built from the merging of NMOS and PMOS, leading to better performances [9]. This transmission gate has high speed due to low input capacitance but it has only limited capacity to drive a load. the advantage of the transmission gate is simple and fast.

The static and transient performance depends upon the availability of high-quality switches with low parasitic resistance and capacitance [10]-[11]. Its static power dissipation is constant and dynamic power is very less. The drawback of transmission gate is that it requires inverted logic to control gates of NMOS and PMOS, respectively. The problems of transmission gates are they diminishes the output as input passes through multiple stages and no isolation is provided between output and input terminal. Figure- 1 shows the symbol of the transmission gate and Figure-2 shows Schematic. When node A has high Logic 1, then complementary $\bar{A}$ has Logic zero is applied on A node, allowing both transistors to conduct and pass the signal between IN to OUT.

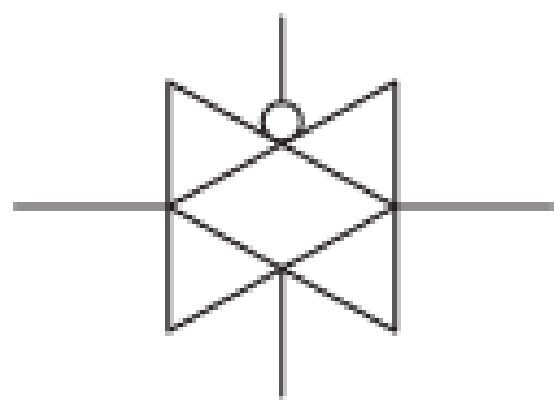

Figure-1 Transmission Gate Symbol 


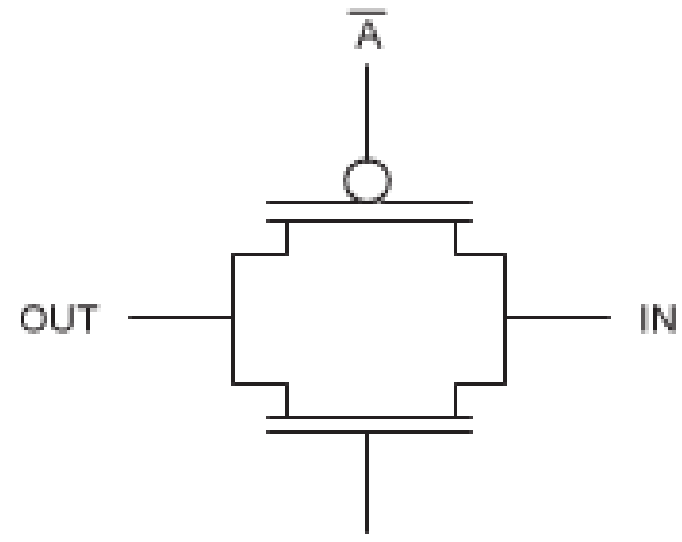

A

Figure-2 Transmission Gate Schematic

\section{PROPOSED SCHEMATIC DESIGN}

In this paper, designing of XOR-XNOR circuit by using a transmission gate inverter circuit in $45 \mathrm{~nm}$ and compare its results with $65 \mathrm{~nm}$ technology. Figure-3 Schematic of XNOR-XOR gate transmission gates. The CMOS inverter is required for the transmission gate to attain the good output voltage swing. A and $\bar{A}$ are provided as the input to transmission gates through CMOS inverter. The output of transmission gates provides the XNOR output and using an inverter we got the XOR output.

The transmission gate allows to passes the signal through it when the enable signal of transmission gate is at logic " 1 ". The transmission gate has a p-channel device and n-channel device, $n$-channel MOS is located on the bottom of the p-channel MOS. When "0" signals logic applies to enable pin the transmission gate is turned off, and no signal is passes from it. When the enable is asserted high, the input signal passes to the output.

Table 1. Shows the truth table for XNOR-XOR gates. Figure-4 Timing Diagrams out1 is out-put of XNOR gate and out 2 is for XOR.

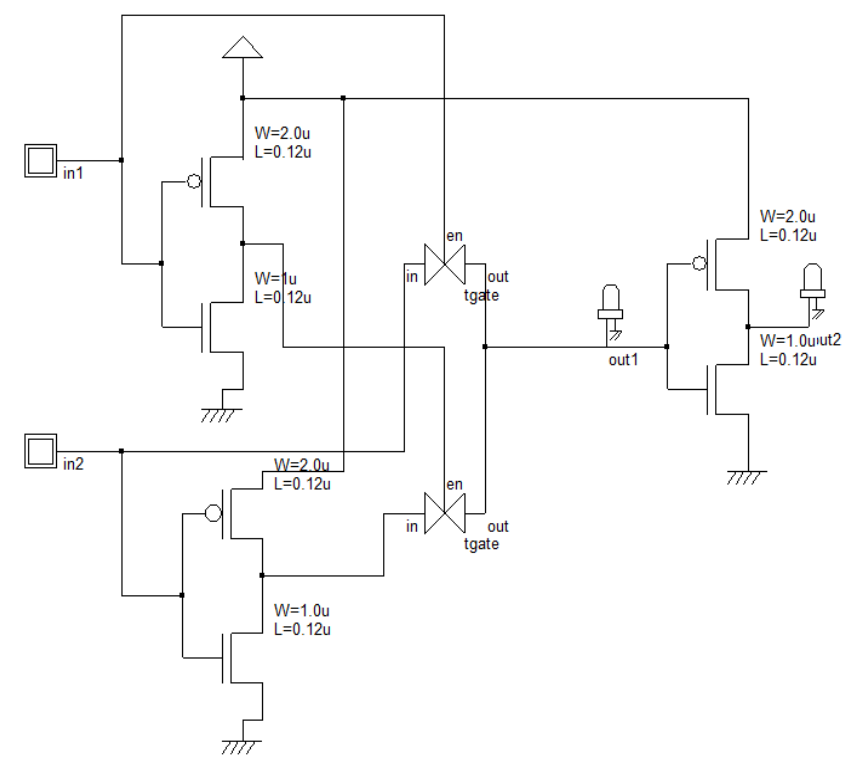

Figure-3 Transmission Gate based Schematic of XNOR-XOR
Table 1 XNOR-XOR Truth Table

\begin{tabular}{|c|c|c|c|}
\hline in1 & in2 & XNOR & XOR \\
\hline 0 & 0 & 1 & 0 \\
\hline 0 & 1 & 0 & 1 \\
\hline 1 & 0 & 0 & 1 \\
\hline 1 & 1 & 1 & 0 \\
\hline
\end{tabular}

clk1

clk2

out1

out2

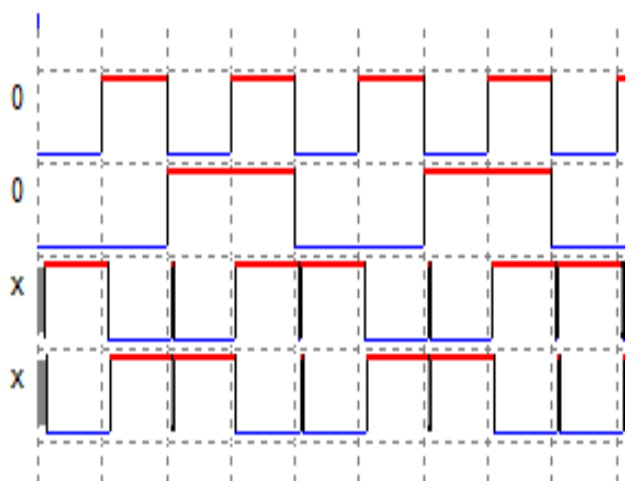

Figure-4 Schematic Simulation

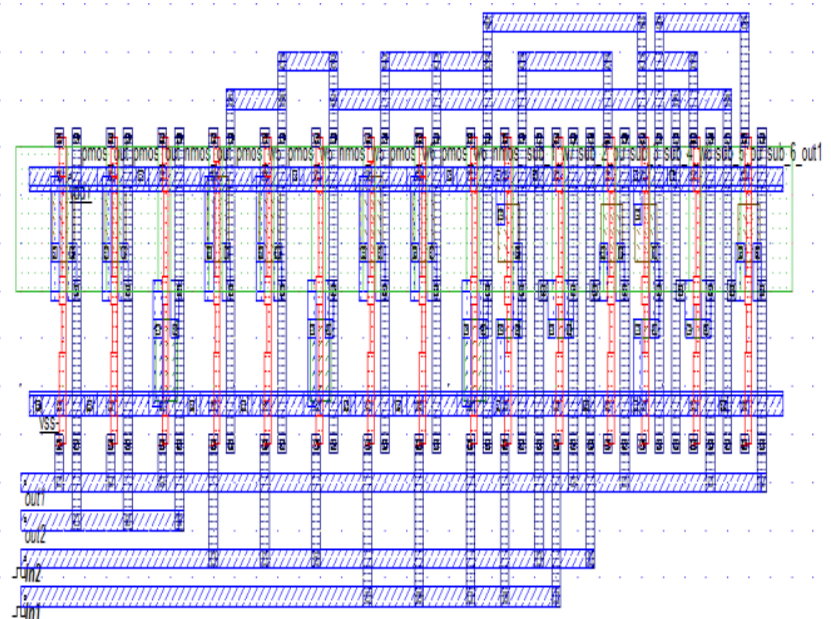

Figure-5 Layout Design- XNOR-XOR with Transmission

\section{LAYOUT DESIGN ANALYSIS}

Once the circuit designing is completed in Dsch2 tool then Verilog file is created for circuit. Files is compiled in Microwind3.1 for layout design and simulation. Figure-5 shows layout design for XNOR-XOR circuit using transmission gate.

The XNOR-XOR gate circuit is simulated in Microwing3.1 with $45 \mathrm{~nm}$ technology. Verilog files of designed circuit is compiled in Microwind3.1 with foundry setting in $45 \mathrm{~nm}$. XNOR-XOR using transmission gate (TG) are and simulation results is shown in Figure-6 and Figure-7 respectively for $45 \mathrm{~nm}$ technology. The analog simulation has been performed and its 


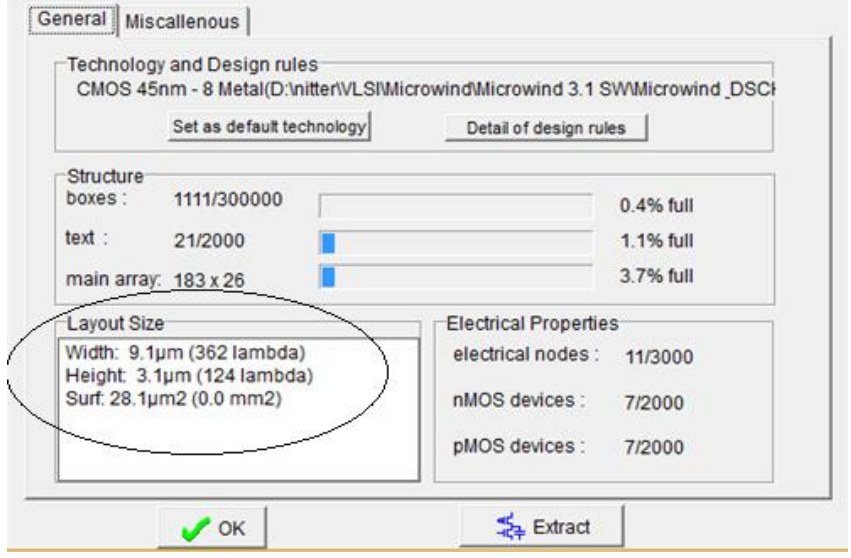

Figure-6 Total Area - XNOR-XOR using TG (45nm)

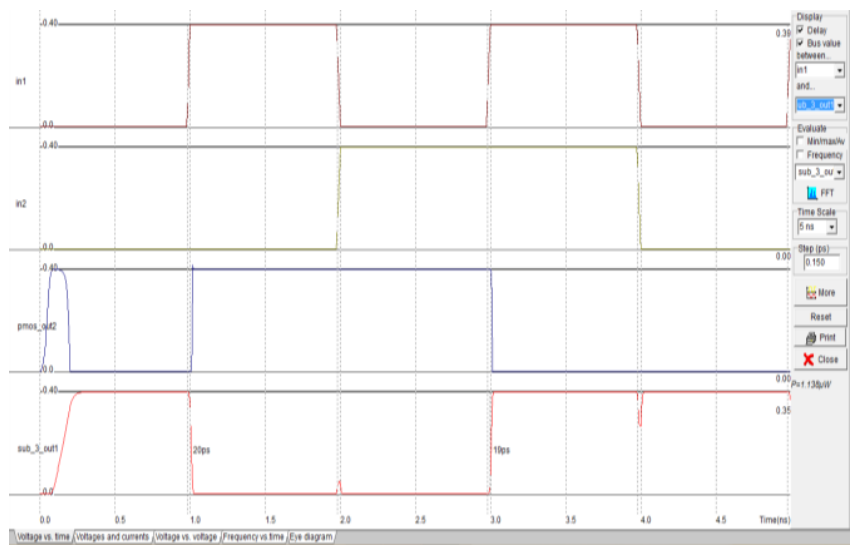

Figure-7 Layout Simulation

Table-2 Results Analysis

\begin{tabular}{|l|c|}
\hline Parameters & Proposed Design \\
\hline $\begin{array}{l}\text { Power Consumption } \\
(\mathrm{uW})\end{array}$ & 1.139 \\
\hline Area (um2) & 28.1 \\
\hline Delay (Ps) & 19 \\
\hline
\end{tabular}

results in term of power, area and speed are shown in Table-2. It shows that XNOR-XOR implementation using transmission gate technology is much better than XNOR-XOR implementation using CMOS. The performance of proposed design is Compared with Existing design[1] and Proposed design are shown in Table-3.

Table-3 Results Comparison

\begin{tabular}{|l|c|c|c|}
\hline Parameter & $\begin{array}{l}\text { Existing } \\
\text { Design } \\
{[1]}\end{array}$ & $\begin{array}{l}\text { Proposed } \\
\text { Design }\end{array}$ & $\begin{array}{l}\text { Improvement } \\
(\boldsymbol{\%})\end{array}$ \\
\hline $\begin{array}{l}\text { Power } \\
\text { Consumption } \\
(\mathbf{u W})\end{array}$ & 12.198 & 1.139 & 90.66 \\
\hline Area (um2) & 41.7 & 28.1 & 63.97 \\
\hline $\begin{array}{l}\text { Propagation } \\
\text { Delay (Ps) }\end{array}$ & 130 & 19 & 85.38 \\
\hline
\end{tabular}

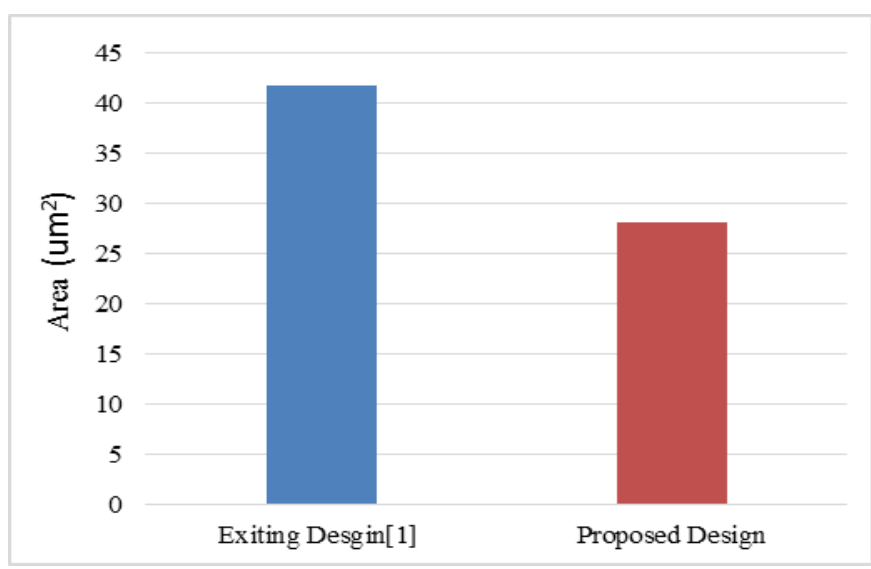

Figure-8 Area Required

Figure- 8 shows the area graph in both designs technologies. It shows that for implementation of XNOR-XOR using transmission gate required $28.1 \mathrm{um}^{2}$ area and exiting design required $41.7 \mathrm{um}^{2}$ area.

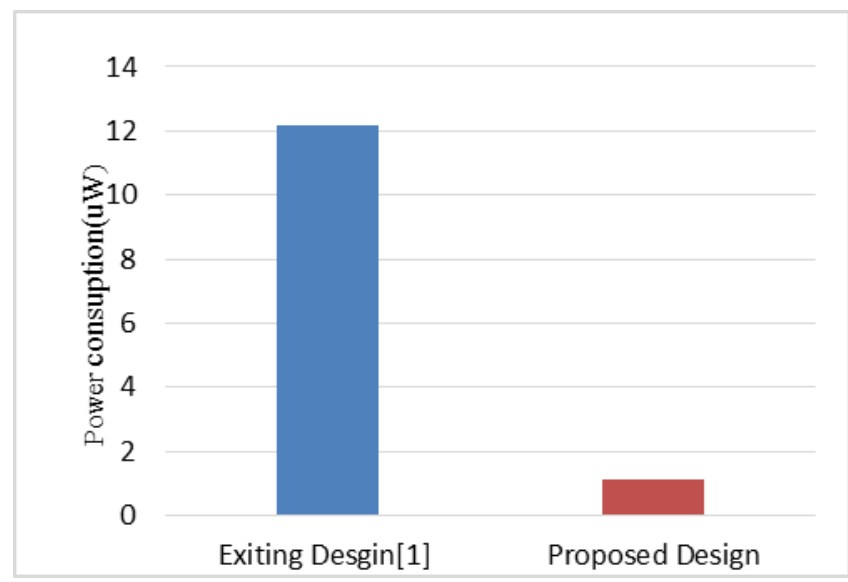

Figure-9 Power Consumption(uW)

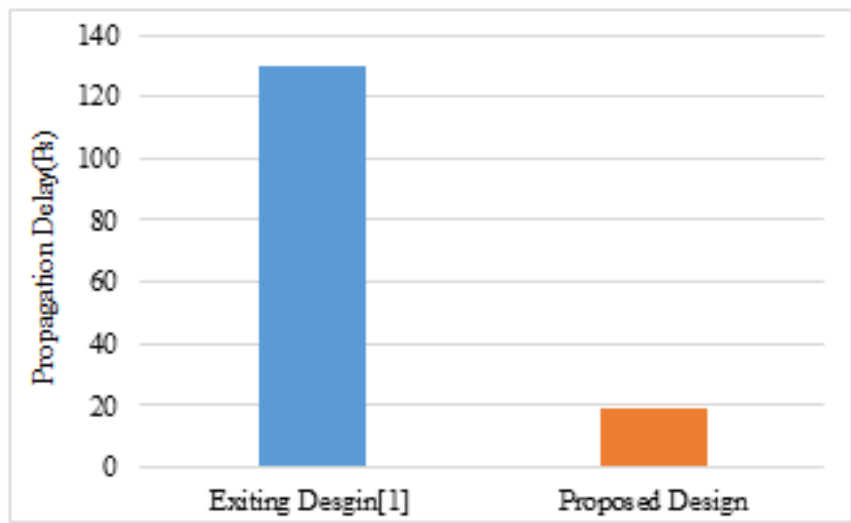

Figure-10 Propagation delay(Ps)

Figure-9 shows power consumption plots in $\mathrm{uW}$ for XNOR-XOR using transmission gate. Power consumption in XNOR-XOR using CMOS transmission gate consume $1.139 \mathrm{uW}, 12.198 \mathrm{uW}$ in proposed design and exiting design respectively. Figure-10 shows propagation delay in (Ps) for XNOR-XOR using transmission gate for proposed design and existing design. Which is 130ps for existing design and 19ps for proposed design. 


\section{CONCLUSION}

Proposed XNOR-XOR using CMOS transmission gate is designed and simulated successfully in $45 \mathrm{~nm}$ technology. The performance parameters are compared with existing design. From the result it is found that proposed design requires $63.97 \%$ less than existing design. Power consumption in XNOR-XOR using transmission gate consume $90.66 \%$ less in proposed design. Speed of proposed design is $85.38 \%$ fester then exiting design. The importance of the work or suggest applications and extensions.

\section{REFERENCES}

[1] Swati Sharma, Rajesh Mehra "Area \& Power Efficient Design of XNOR-XOR Logic Using 65nm Technology” International Journal of Engineering and Technical Research pp.57-60, 2014.

[2]Nabihah Ahmad, Rezaul Hasan "A New Design of XOR-XNOR gates for low power applications", International Conference on Electronic Devices, System and Applications, pp.45-49, 2011.

[3]W. Jyh-Ming, F. Sung-Chuan, and F. Wu-Shiung, "New efficient designs for XOR and XNOR functions on the transistor level," IEEE Journal of Solid State Circuits, Vol.29, pp. 780-786,1994.

[4]S. Goel, M.A. Elgamel, M.A. Bayoumi and Y. Hanafy "Design Methodologies for High- Performance Noise-Tolerant XOR-XNOR Circuits Design", IEEE Transaction on Circuits and System I: Regular Papers, Vol. 53, pp. 867-878, 2006.

[5]Rajeev Kumar Vimal Kant Pandey, IEEE "A New 5 Transistor XOR-XNOR Circuit based on the Pass Transistor logic "World Conference on Information and communication Technologies, pp-667-671,2011.

[6]S. W. Shiv Shankar Mishra, R. RK. Nagaria and S. Tivari, "New Design Methodologies for High Speed Low Power XOR-XNOR Circuits, "International Journal of Electrical, Computer, Energetic, Electronics and Communication Engineering, Vol: 3, pp. 1427-1433, 2009.

[7]K. E. Neil, H. E. Weste, "Principles of CMOS VLSI Design: A Systems Perspective". 1993, pp. 169-172.

[8]D. Radhakrishnan, "Low-voltage low-power CMOS full adder,"IEE Proceedings- Circuits Devices and Systems, Vol. 148, pp. 19-24, 2001.

[9]M. Elgamel, S. Goel, and M. Bayoumi, "Noise tolerant low voltage XOR-XNOR for fast arithmetic", Conference on Proceedings of the 13th ACM Great Lakes symposium on VLSI, pp. 285-288, 2003.

[10] Tooraj Nikoubin, Mahdieh Grailoo and Changzhi Li, "Energy and Area Efficient Three-Input XOR/XNORs With Systematic Cell Design Methodology", IEEE Transactions on Very Large Scale Integration Systems, Vol: 24, Issue: 1, Jan. 2016.

[11] Neeraj Jain, Puran Gour and Brahmo Shaeman, "A high speed low power adder in dynamic logic base on transmission gate", International Conference on Circuits, Power and Computing Technologies, pp. 1-5, 2015.

[12] Zhixi Yang, Jie Han and Fabrizio Lombardi, "Transmission gate based approximate adders for inexact computing", Proceeding of the 2015 IEEE/ACM international Symposium on Nanoscale Architectures, pp. 145-150, 2015.

[13] Neeraj Jain, Puran Gour, Brahmi Shrman, "A High speed Low Power Adder in Multi Output Domino Logic", International Journal of Computer Applications, vol. 105, no. 7, pp. 0975-8887, November 2014 